\title{
Terahertz imaging: a new non-destructive technique for the quality control of plastic weld joints
}

\author{
S. Wietzke \\ steffen.wietzke@inf.tu-bs.de
}

C. Jördens

\section{N. Krumbholz}

\section{B. Baudrit}

\section{Bastian}

M. Koch

\begin{abstract}
Institut für Hochfrequenztechnik, Technische Universität Braunschweig, Schleinitzstraße 22, 38106 Braunschweig, Germany Joint Optical Metrology Center (JOMC) c/o Technische Universität Braunschweig, Fakultät für Elektrotechnik und Informationstechnik, Hans-Sommer-Straße 66, 38106 Braunschweig, Germany Institut für Hochfrequenztechnik, Technische Universität Braunschweig, Schleinitzstraße 22, 38106 Braunschweig, Germany

Joint Optical Metrology Center (JOMC) c/o Technische Universität Braunschweig, Fakultät für Elektrotechnik und Informationstechnik, Hans-Sommer-Straße 66, 38106 Braunschweig, Germany Institut für Hochfrequenztechnik, Technische Universität Braunschweig, Schleinitzstraße 22, 38106 Braunschweig, Germany Joint Optical Metrology Center (JOMC) c/o Technische Universität Braunschweig, Fakultät für Elektrotechnik und Informationstechnik, Hans-Sommer-Straße 66, 38106 Braunschweig, Germany
\end{abstract} Süddeutsches Kunststoff-Zentrum KFE, Friedrich Bergius-Ring 22, 97076 Würzburg, Germany Süddeutsches Kunststoff-Zentrum KFE, Friedrich Bergius-Ring 22, 97076 Würzburg, Germany

Institut für Hochfrequenztechnik, Technische Universität Braunschweig, Schleinitzstraße 22, 38106 Braunschweig, Germany

Joint Optical Metrology Center (JOMC) c/o Technische Universität Braunschweig, Fakultät für Elektrotechnik und Informationstechnik, Hans-Sommer-Straße 66, 38106 Braunschweig, Germany

We present the first investigation of plastic weld joints using terahertz waves. Terahertz time-domain spectroscopy clearly reveals contaminations like metal or sand within the weld joint of two high-density polyethylene sheets. Furthermore, areas can be identified where the welding process has failed and the parts to be joined are separated by a small air gap. We show that a three layer structure of polyethylene-air-polyethylene has a characteristic, frequency-dependent transmission behaviour. This allows for a distinction between welded and non-welded material as well as for the calculation of the air layer thickness from the relative transmission spectrum. Consequently, terahertz time-domain spectroscopy provides a promising new non-destructive and even contactless technique, which is desired by the plastics industry for detecting a variety of deviations from the ideal welding process. [D0I: 10.2971/je0s.2007.07013]

Keywords: Terahertz imaging, non-destructive testing, quality control, weld joint, plastics

\section{INTRODUCTION}

As a rather young group of materials, plastics have experienced a rapid development during the last decades. Highly specific mechanical parameters (stiffness and strength in terms of the specific gravity), corrosion resistance and the compatibility with inexpensive machining like extrusion or injection molding paved their way to applications ranging from commodities to high tech products [1]. Furthermore, compounding of standard polymers with a wide range of additives makes it possible to specifically adjust the component properties to the respective product requirements.

Recently, plastics have also gained importance as construction materials. This draws the attention to joining methods such as welding, adhesive bonding and mechanical joining. With welding, the polymeric material is softened or melted at the component interfaces to be joined so that new molecular chain entanglements let the parts stick together [1]. Contami- nations, air bubbles or delaminations can reduce the strength of the weld dramatically, eventually leading to a loss of structural integrity. For instance, the weld between HDPE pipes used in gas, water and disposal systems requires a thorough quality control in order to avoid potentially dangerous leaks. Since welding is normally placed at the end of the value creation chain, ensuring the quality of plastic weld joints is also important for non-structural applications due to economical reasons.

The commonly used methods for weld quality inspection are application specific [2]. They depend on the chosen welding method and joint design as well as on the typical mechanical load on the component. Widely used mechanical techniques, e.g. the tensile or bend test, yield quantitative results. They are standardized but restricted to certain part geometries and load cases [3]. Moreover, these testing methods require prepa- 
ration time and they are destructive techniques. However, especially in the case of the HDPE pipe welds mentioned above, a reliable evaluation can so far be achieved only by destructive testing.

All attempts to find a non-destructive testing method for weld quality control have yielded unsatisfying results or the test methods are economically inefficient. Visual inspection, for example, requires an experienced inspector in order to discover all imperfections of the weld joint [2]. X-ray testing is not suited for mass production but rather for special products with high safety requirements. Ultrasonic testing is capable of the detection of air bubbles and voids as well as contaminations but there are restrictions concerning the maximum material thickness: it is compatible only with certain polymers because of the relatively high attenuation in thermoplastics [2]. The non-destructive quality control is hence most times restricted to a preventive monitoring of the welding process parameters [4].

In this paper, we evaluate the potential of terahertz $(\mathrm{THz})$ imaging for non-destructive testing of plastic weld joints. First images obtained with an optoelectronic $\mathrm{THz}$ system were presented in 1995 by Hu and Nuss [5]. These THz images reveal the structure of an integrated circuit beneath its black plastic package or visualize the water distribution in a leaf. Since then, a multitude of applications, especially in the area of nondestructive testing, has been discussed [6]-[10]. In particular in the plastics industry, many applications for $\mathrm{THz}$ time-domain spectroscopy (THz TDS) are foreseen [11, 12], as most standard polymers are transparent in the lower $\mathrm{THz}$ frequency range, i.e at frequencies between $100 \mathrm{GHz}$ and a few THz [13].

In the following, we present first results of $\mathrm{THz}$ measurements on plastic samples with differing weld quality. As expected, inclusions of sand and metal between two plastic sheets can clearly be discovered in a THz TDS image due to scattering and absorption (sand), and reflection (metal). Furthermore, $\mathrm{THz}$ TDS is capable of detecting the end of a weld contact area. Moreover, the thickness of an air layer between two non-welded plastic sheets can be calculated from the relative transmission spectrum. Hence, THz TDS imaging is a highlyvaluable approach to develop a non-destructive and even contactless testing method to monitor the weld quality of plastic components - an attainment, long sought after by the plastics industry and their respective organizations.

\section{SAMPLES AND EXPERIMENT}

$\mathrm{THz}$ imaging allows for an investigation of many samples which are opaque in the visible. However, in order to obtain a visual verification of the $\mathrm{THz}$ data, we choose here a highdensity polyethylene (HDPE) from Simona AG, Kirn, Germany, which is translucent in the visible and has a refractive index of $n=1.54$ in the $\mathrm{THz}$ frequency range. Three millimeter thick sheets welded in a lap joint with varying qualities served as samples for the measurements. A variety of deviations from the ideal welding process like contaminations by sand or metal items, here for instance staples, were introduced. Furthermore, one sample with a partial welding was studied to investigate whether $\mathrm{THz}$ imaging reveals a contrast between areas of full material penetration and areas in which the two sheets simply touch each other or are separated by a small air gap.

Transmission measurements were performed on the prepared samples using a standard THz TDS setup with photoconductive dipole antennas excited by femtosecond pulses from a titanium:sapphire laser. The THz TDS setup is in detail described elsewhere [14]. To obtain images, the samples are placed in the intermediate focus of the setup perpendicular to the $\mathrm{THz}$ beam and scanned in a raster pattern in two dimensions [6]. The step width is $\Delta x=\Delta y=0.5 \mathrm{~mm}$. At each position, the waveforms of five $\mathrm{THz}$ pulses having propagated through the sample are acquired and averaged. In a consecutive step, a fast Fourier transform of the THz pulse in the timedomain is calculated. For each pixel, the $\mathrm{THz}$ pulses are analyzed. Different features of the time-domain data or the phase and amplitude information of the corresponding Fourier spectra can be plotted - each feature leading to a different image [15]. These images provide information about the composition and the structure of the sample under investigation [15]. Here we focus on the intensity data, integrated over a certain frequency window. Later on, we will show that this analysis mode is well suited for the applications proposed in this paper.

\section{RESULTS AND DISCUSSION}

If the dielectric $\mathrm{THz}$ properties of the inclusions differ from those of the polymer the contaminations within the weld joint should be easily detectable. This is the case for both metal and sand.

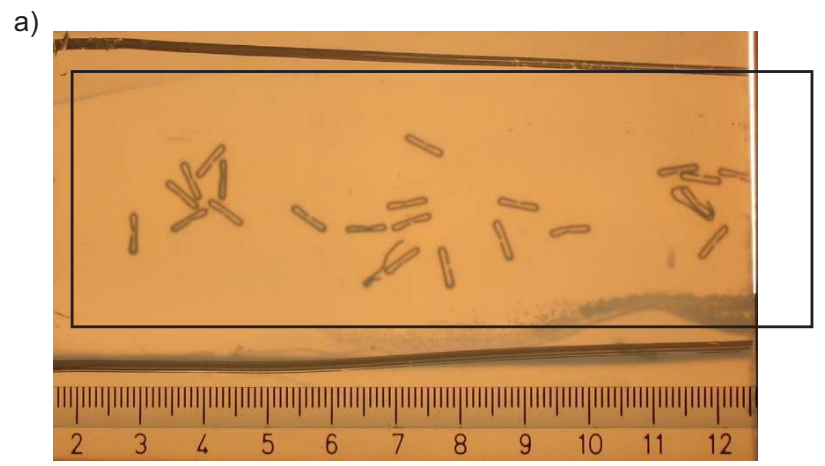

b) Normalized intensity

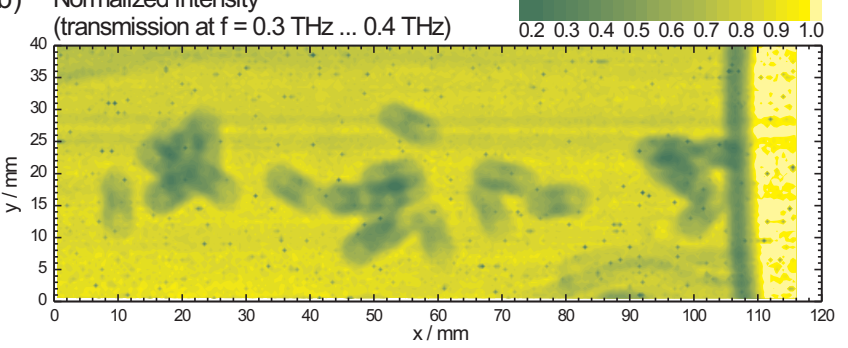

FIG. 1 Photograph of the back-lighted sample with metal staples within the weld contact area (a). The THz TDS image (b) displaying the transmitted intensity integrated from 0.3 THz to 0.4 THz shows the analyzed region of the sample marked by the black frame: The staples can clearly be detected due to their high extinction coefficient. 
Figure 1a shows a photograph of the back-lighted sample sheets which contain metal staples in the weld contact area. The black frame indicates the area which is investigated by $\mathrm{THz}$ imaging. The white stripe on the very right corresponds to a free-space area which is included in the measurements in order to correct the sample amplitude for long term drifts of the laser power.

Figure $1 \mathrm{~b}$ shows the corresponding $\mathrm{THz}$ transmission image (normalized intensity) obtained for the frequency range from $0.3 \mathrm{THz}$ to $0.4 \mathrm{THz}$. This frequency range is a compromise between good spatial resolution and high signal-to-noise ratio. The high extinction coefficient of metal makes it easy to identify the individual metal staples in the $\mathrm{THz}$ image of the weld joint as areas of low transmission.

a)

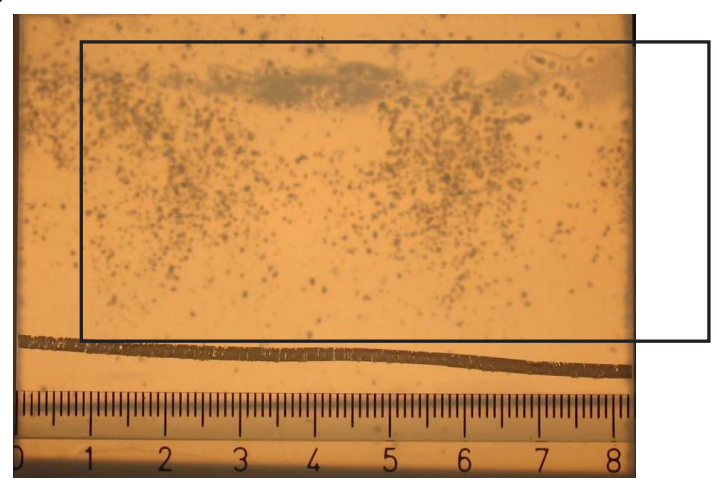

b) Normalized intensity

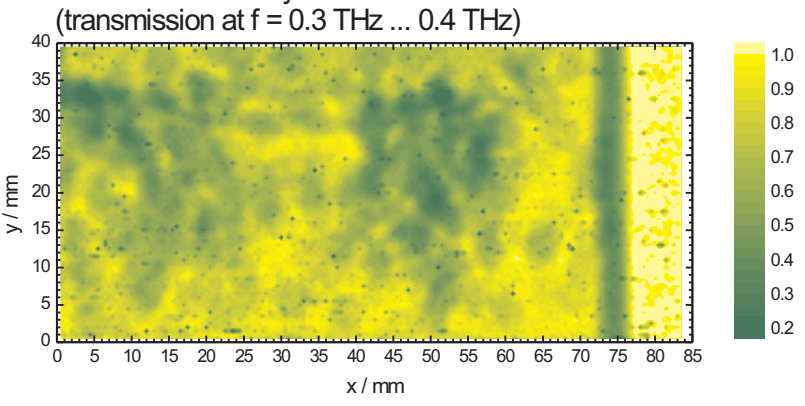

FIG. 2 Photograph of the back-lighted sample with sand contaminating the weld joint (a). The THz TDS image (b) taken from the marked region displays the transmitted intensity integrated from $0.3 \mathrm{THz}$ to $0.4 \mathrm{THz}$. Due to the limited spatial resolution, individual sand grains cannot be resolved, but cloudy areas of lower transmission reveal the clusters of a large number of particles.

Their structure cannot be resolved exactly because the spatial resolution is limited by the wavelengths employed. The vertical stripe of low transmission on the right hand side results from diffraction at the sample edge $[15,16]$. The bright horizontal line around $y=27 \mathrm{~mm}$ is a measurement artefact. Single pixels representing reduced transmission arise from laser instability.

Also contaminations by sand can be detected. While Figure 2a shows the visible image, Figure $2 b$ depicts the transmitted intensity between $0.3 \mathrm{THz}$ to $0.4 \mathrm{THz}$.

Individual sand grains cannot be resolved but clusters of a large number of particles appear as cloudy structures. This is mainly due to scattering as well as due to their higher absorption compared with HDPE. Both result in a lower transmission.

Aside from visualizing inclusions, THz TDS is also capable of displaying the end of a weld contact area. In the picture of the only partially welded sample sheets (Figure 3a) a distinctive line (marked by the arrow) can be seen. It marks the border of the proper lap joint (above) and the area where the HDPE sheets are separated by a small air gap (below). The THz TDS image (Figure $3 b$ ) clearly reproduces this feature: In the lower left area, there is a noticeable stripe of high transmission from $\left(x_{1} ; y_{1}\right)=(0 ; 10 \mathrm{~mm})$ to $\left(x_{2} ; y_{2}\right)=(50 \mathrm{~mm} ; 0)$ which is a typical signature of diffraction off an edge $[15,16]$.

a)

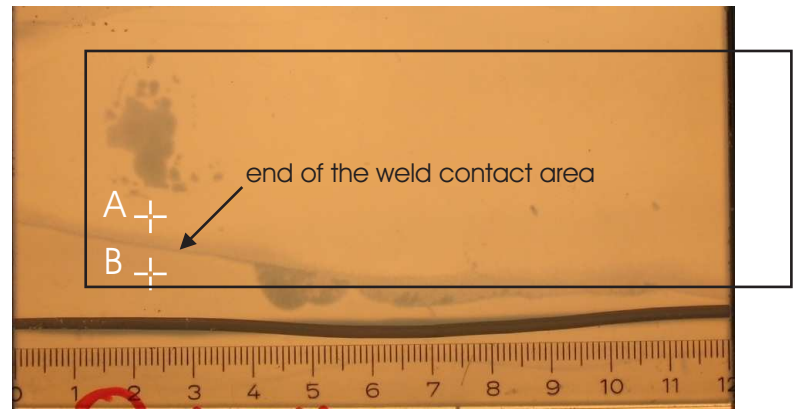

b)

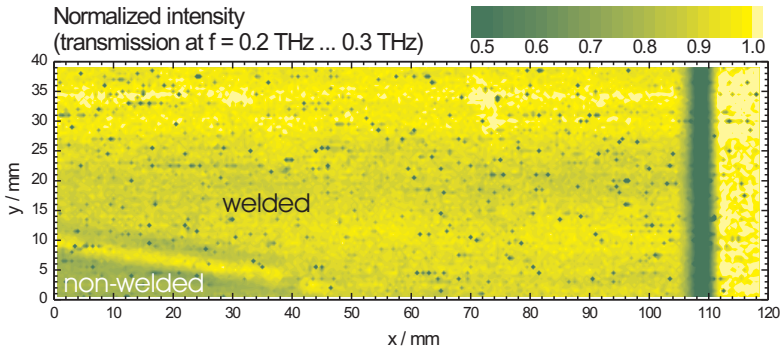

FIG. 3 Photograph of the back-lighted sample which is only partially welded (a). The THz TDS image (b) of the region marked by the black frame shows the transmitted intensity integrated from $0.2 \mathrm{THz}$ to $0.3 \mathrm{THz}$. It clearly reproduces the end of the weld contact area. Below the stripe from $\left(x_{1} ; y_{1}\right)=(0 ; 10 \mathrm{~mm})$ to $\left(x_{2} ; y_{2}\right)=(50 \mathrm{~mm} ; 0)$, the HDPE sheets are not welded.

Below, an area of significantly decreased transmission reveals that the sheets are not welded. Since polyethylene hardly absorbs in the far infrared, fluctuations in material thickness resulting from the welding process are supposed to have negligible influence on the $\mathrm{THz}$ signal.

However, the difference in transmission between welded and non-welded material is clearly visible in the THz image showing the transmitted intensity integrated between $0.2 \mathrm{THz}$ and $0.3 \mathrm{THz}$. This finding can be explained when turning to the $\mathrm{THz}$ pulses acquired in these two regions of the sample. In Figure 3a, two positions are marked: one for the case that the material is well welded (A) and one in the area without a weld joint (B). The pulses are plotted in Figure 4. as solid and dashed line, respectively. Although the two pulses look similar to each other at the first glance some differences can be noticed e.g. around $t=21 \mathrm{ps}$ (Figure 4 ).

They arise from a small Fabry-Pérot reflection which superim- 


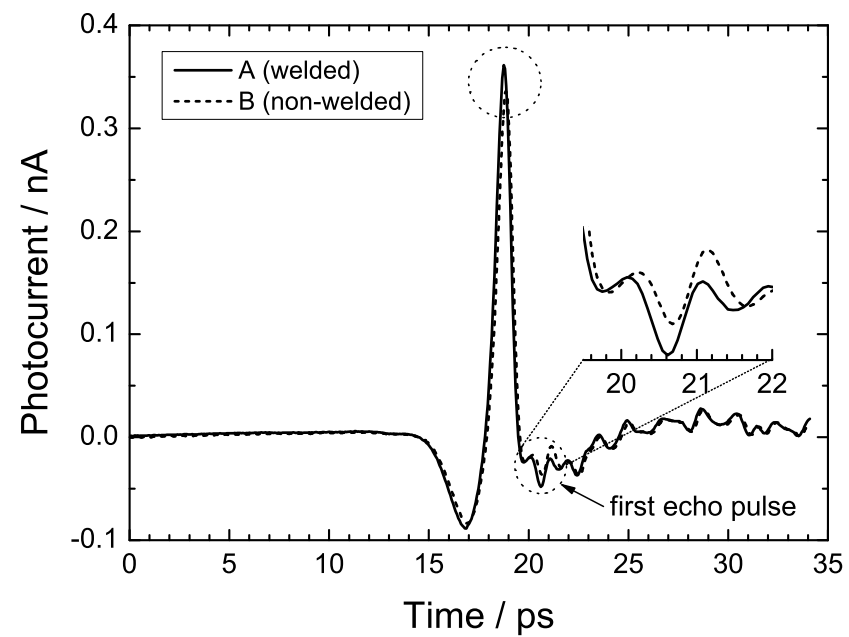

FIG. 4 THz pulses through a welded ( - solid line) and a non-welded ( $B$ - dashed line) part of the sample. The positions A, B can be seen in Figure $3 a$. The circled area indicates the temporal position of the first reflection within the intermediate air layer at position B. This echo pulse superimposes the oscillations due to water absorption.

poses the oscillations due to water vapour which follow the main pulse. Subsequent Fabry-Pérot echoes are too small to be seen in this plot.

The Fabry-Pérot reflections lead to a nearly sinusoidal shape in the relative transmission. This can be seen in Figure 5 (solid line) where we plot the spectrum of pulse B divided by the spectrum of pulse A. The modulated transmission is easy to understand as a layer with a thickness $d$ in between two dielectric sheets leads to enhanced reflection and reduced transmission at wavelengths which correspond to $d=p \lambda / 4$, with $p=1,3,5, \ldots$. At wavelengths corresponding to $d=q \lambda / 2$ with $q=1,2,3, \ldots$ maximum transmission through the structure is observed.

The sinusoidal shape can be reproduced by a simulation which takes into account a number of Fabry-Pérot reflections. In the simulation, the result of which is shown in Figure 5 as a dashed line, we assume a HDPE-air-HDPE structure with an air layer thickness of $320 \mu \mathrm{m}$ and include eight echo pulses. This air layer thickness corresponds to $\lambda / 4$ and $3 / 4 \lambda$ for frequencies of $234 \mathrm{GHz}$ and $703 \mathrm{GHz}$, respectively. The simulation result fits to the measured data and emphasizes the sinusoidal oscillation of the measured relative transmission. In Figure 5, there are also two maxima of relative transmission around $469 \mathrm{GHz}$ and $938 \mathrm{GHz}$.

The overall decrease in transmission with rising frequency which is observed in the experiment is caused by increasing scattering losses for higher frequencies at the two additional interfaces. This frequency-dependent transmission explains why the deviation between welded and non-welded material in the transmission image is best seen between 0.2 and $0.3 \mathrm{THz}$ (Figure $3 \mathrm{~b}$ ).

In principle, it should be no problem to detect air gaps which are much smaller than $320 \mu \mathrm{m}$. Yet, the first minimum in the relative transmission will occur at higher frequencies if the air layer is thinner. This is illustrated in Figure 6, which shows

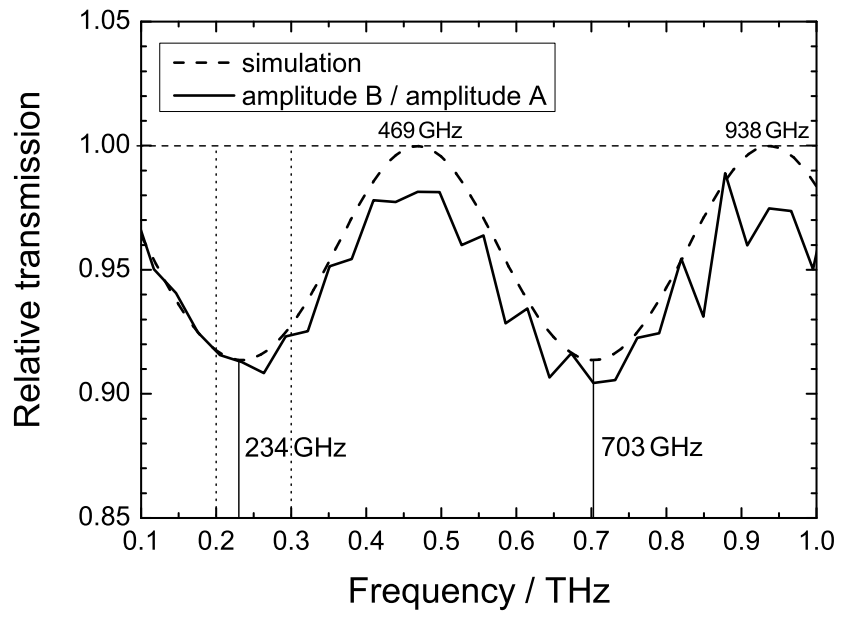

FIG. 5 Relative transmission, i.e. amplitude B divided by amplitude A, between $0.1 \mathrm{THz}$ and 1.0 THz: A simulation assuming an air layer of $d=320 \mu \mathrm{m}$ between the two HDPE sheets confirms a characteristic sinusoidal oscillation of the measured data. The spectral positions of the maxima and minima allow for calculating the thickness of the air gap.

simulations for air layers of $d=320 \mu \mathrm{m}, d=50 \mu \mathrm{m}$ and $d=10 \mu \mathrm{m}$. Hence, the detection limit regarding the lowest layer thickness depends on the system, i.e. on its signal stability, bandwidth, and signal to noise ratio. Recently, we demonstrated a system with excellent long term amplitude stability. It is based on a $1.55 \mu \mathrm{m}$ fibre laser and low-temperaturegrown InGaAs photoconductive antennas [17].

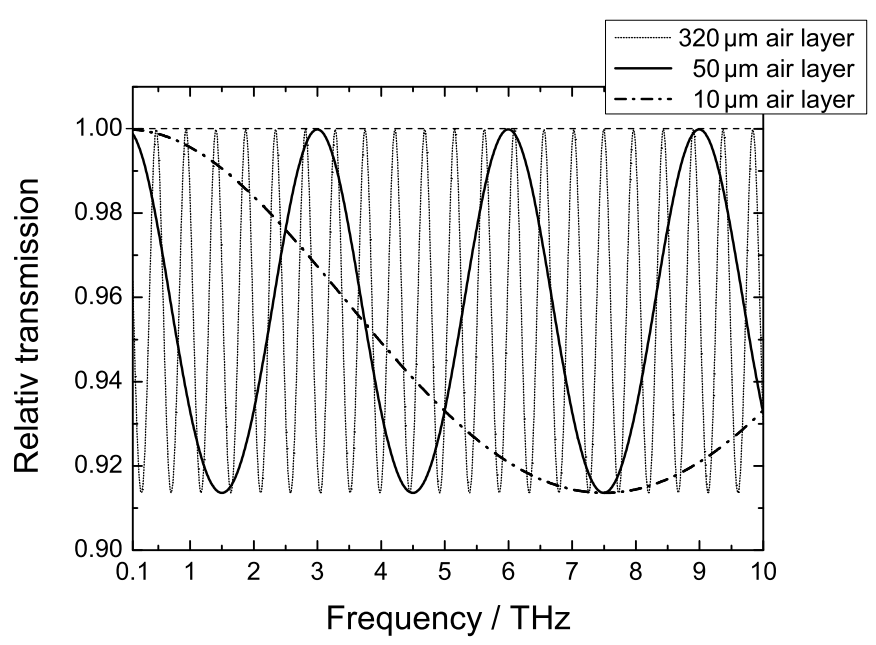

FIG. 6 Simulations of the frequency-dependent transmission spectrum for air layers of $d=320 \mu \mathrm{m}, d=50 \mu \mathrm{m}$ and $d=10 \mu \mathrm{m}$ : The lower the size of the layer is, the larger becomes the distance between the odd multiples of the $\lambda / 4$ frequencies.

Since the integration window should be centred around the first relative transmission minimum or at least be located in a frequency region where the relative transmission deviates noticeably from one, very thin air layers call for images obtained at several THz. Here, systems based on quantum cascade lasers might have to be used to supply these operation frequencies. 


\section{CONCLUSIONS}

In summary, we have shown that $\mathrm{THz}$ TDS imaging is a promising new technique for the quality control of plastic weld joints. We have investigated several high-density polyethylene sheets welded in a lap joint with different welding imperfections. Contaminations of metal or sand within the weld joint of two HDPE sheets can clearly be located. Moreover, THz TDS can distinguish between welded and nonwelded material. The thickness of an air layer between two non-welded plastic sheets can be calculated. For the investigated sample it is determined to be $d=320 \mu \mathrm{m}$. Consequently, $\mathrm{THz}$ TDS is capable of detecting a variety of deviations from the ideal welding process like contaminations or delamination.

\section{ACKN OWLEDGEMENT}

We thank C. Jansen for careful reading of the manuscript. Furthermore, we acknowledge the Federal Ministry of Education and Research for funding us within the frame of the joint project "Femtonik" (FKZ: 13N8572).

\section{References}

[1] A. Benatar, "Introduction" in Plastics and Composites Welding Handbook, D. A. Grewell, A. Benatar, and J. B. Park, eds., 1-9 (Hanser, Munich, 2003).

[2] C. Bonten and C. Tuechert, "Testing of weld joints" in Plastics and Composites Welding Handbook, D. A. Grewell, A. Benatar, and J. B. Park, eds., 361-392 (Hanser, Munich, 2003).

[3] N. N., DVS 2203: Prüfen von Schweißverbindungen aus thermoplastischen Kunststoffen“ (DVS, Düsseldorf, 1985-1999).

[4] E. Pecha and A. Savitski, "Heated tool (hot plate) welding" in Plastics and Composites Welding Handbook, D. A. Grewell, A. Benatar, and J. B. Park, eds., 29-71 (Hanser, Munich, 2003).

[5] B. B. Hu and M. C. Nuss, "Imaging with terahertz waves" Opt. Lett. 20, 1716-1718 (1995).
[6] S. Hunsche, D. M. Mittleman, M. Koch, and M. C. Nuss, "New dimensions in t-ray imaging" IEICE T. Electron. E81-C, 269-276 (1998).

[7] K. Kawase, Y. Ogawa, and Y. Watanabe, "Non-destructive terahertz imaging of illicit drugs using spectral fingerprints" Opt. Express 11, 2549-2554 (2003).

[8] N. Karpowicz, H. Zhong, C. Zhang, K.-I. Lin, J.-S. Hwang, J. Xu, and X.-C. Zhang, "Compact continuous-wave subterahertz system for inspection applications" Appl. Phys. Lett. 86, 054105 (2005).

[9] T. Kiwa, M. Tonouchi, M. Yamashita, and K. Kawase, "Laser terahertz-emission microscope for inspecting electrical faults in integrated circuits" Opt. Lett. 28, 2058-2060 (2003).

[10] T. Yasui, T. Yasuda, K. Sawanaka, and T. Araki, "Terahertz paintmeter for noncontact monitoring of thickness and drying progress in paint film" Appl. Opt. 44, 6849-6856 (2005).

[11] F. Rutz, M. Koch, S. Khare, M. Moneke, H. Richter, and U. Ewert, "Terahertz quality control of polymeric products" Int. J. Infrared Milli. 27, 547-556 (2006).

[12] F. Rutz, T. Hasek, M. Koch, H. Richter, and U. Ewert, "Terahertz birefringence of liquid crystal polymers" Appl. Phys. Lett. 89, 221911 (2006).

[13] Y. S. Jin, G. J. Kim, and S. G. Jeon, “Terahertz dielectric properties of polymers" J. Korean Phys. Soc. 49, 513-517 (2006).

[14] D. M. Mittleman, ed., Sensing with Terahertz Radiation (Springer, Berlin/Heidelberg, 2003).

[15] M. Herrmann, M. Tani, and K. Sakai, "Display modes in timeresolved terahertz imaging" Jpn. J. Appl. Phys. 1 39, 6254-6258 (2000).

[16] S. Hunsche, M. Koch, I. Brener, and M.C. Nuss, "THz near-field imaging" Opt. Commun. 150, 22 (1998).

[17] R. Wilk, M. Mikulics, K. Biermann, H. Künzel, I. Z. Kozma, R. Holzwarth, B. Sartorius, M. Mei, and M. Koch, "THz time-domain spectrometer based on LT-InGaAs photoconductive antennas exited by a $1.55 \mu \mathrm{m}$ fibre laser" Conference on Lasers and ElectroOptics 2007, Baltimore, Maryland, May 2007. 